\title{
Dopamine and Glutamate Control Area-Restricted Search Behavior in Caenorhabditis elegans
}

\author{
Thomas Hills, Penelope J. Brockie, and Andres V. Maricq \\ Department of Biology, University of Utah, Salt Lake City, Utah 84112-0840
}

\begin{abstract}
Area-restricted search (ARS) is a foraging strategy used by many animals to locate resources. The behavior is characterized by a timedependent reduction in turning frequency after the last resource encounter. This maximizes the time spent in areas in which resources are abundant and extends the search to a larger area when resources become scarce. We demonstrate that dopaminergic and glutamatergic signaling contribute to the neural circuit controlling ARS in the nematode Caenorhabditis elegans. Ablation of dopaminergic neurons eliminated ARS behavior, as did application of the dopamine receptor antagonist raclopride. Furthermore, ARS was affected by mutations in the glutamate receptor subunits GLR-1 and GLR-2 and the EAT-4 glutamate vesicular transporter. Interestingly, preincubation on dopamine restored the behavior in worms with defective dopaminergic signaling, but not in $g l r-1, g l r-2$, or eat- 4 mutants. This suggests that dopaminergic and glutamatergic signaling function in the same pathway to regulate turn frequency. Both GLR-1 and GLR-2 are expressed in the locomotory control circuit that modulates the direction of locomotion in response to sensory stimuli and the duration of forward movement during foraging. We propose a mechanism for ARS in C. elegans in which dopamine, released in response to food, modulates glutamatergic signaling in the locomotory control circuit, thus resulting in an increased turn frequency.
\end{abstract}

Key words: glutamate receptor; dopaminergic signaling; area-restricted search; locomotion; neural circuit; Caenorhabditis elegans; glr-1; glr-2; eat-4; cat-2

\section{Introduction}

For survival, an organism must find resources such as food, mates, and territory. Many species use stereotypic foraging strategies to find essential resources, and thus, the distribution of these resources can influence movement behavior. In turn, movement behavior can influence population ecology, the spread of invasive species, and biodiversity (Slatkin, 1985; Kareiva and Odell, 1987; Andow et al., 1990; Grunbaum, 1998). Although our understanding of the ecological and evolutionary significance of foraging behavior has advanced considerably, the neural and molecular mechanisms underlying this behavior are largely unknown.

One type of foraging strategy that is found in virtually all organisms ranging from insects to vertebrates is the arearestricted search (ARS) (Kareiva and Odell, 1987; Bell, 1991; Benedix, 1993; Keaser et al., 1996; Grunbaum, 1998). Animals using ARS will turn more frequently after an encounter with food, restricting their search around the area where food was last found. As the period of time since the last food encounter increases, animals turn less frequently and begin to move away,

Received June 23, 2003; revised Nov. 25, 2003; accepted Dec. 8, 2003.

This work was supported in part by the National Institutes of Health (NIH) Training Program in Genetics (5T32 GM07464), by National Science Foundation Grant 9876262, and by NIH Grant NS35812. We thank Fred Adler, Jon Seger, Neil Vickers, and Charles Shimp for discussions during the research process and members of the Maricq laboratory for comments on this manuscript. We also thank Robert Nass and Robert Blakely for providing the dat-1 promoter and the Caenorhabditis Genetics Center for C. elegans strains.

Correspondence should be addressed to Dr. Andres V. Maricq, Department of Biology, University of Utah, 257 South 1400 East, Salt Lake City, UT 84112-0840. E-mail: maricq@biology.utah.edu.

DOI:10.1523/JNEUROSCI.1569-03.2004

Copyright $\odot 2004$ Society for Neuroscience $\quad$ 0270-6474/04/241217-09\$15.00/0 following a more linear path. This strategy increases the time spent where food is likely to be found and allows the search to be expanded to new areas when the food supply is exhausted.

Theoretical models predict that ARS has adaptive benefits in environments where food is distributed in clumps (i.e., when food in one location is highly correlated with food at nearby locations) (Kareiva and Odell, 1987; Walsh, 1996; Grunbaum, 2000). These models assume generalized "internal state factors" that modulate turning frequency as a function of the time since food was last encountered. One candidate for an internal state factor is the neurotransmitter dopamine. Dopamine has been shown to act both presynaptically and postsynaptically to modulate synaptic signaling (Cameron and Williams, 1993; Umemiya and Raymond, 1997; Yan et al., 1999; Nicola et al., 2000), which in turn can alter the properties of downstream behavioral rhythms (Barnes et al., 1994; Harris-Warrick et al., 1995). Defects in dopaminergic signaling are also implicated in a wide range of disorders in goal-directed behavior. These include the response to novel stimuli (Hollerman and Schultz, 1998; Dulawa et al., 1999; Zhuang et al., 2001), feeding behaviors (Szczypka et al., 2001), drug addiction (Rocha et al., 1998; Berke and Hyman, 2000), attention deficit hyperactivity disorder (Faraone et al., 2001), Parkinson's disease (Marsden, 1992), and schizophrenia (Hietala and Syvalahti, 1996).

Dopamine can modify the kinetics of ligand-gated ion channels, presumably leading to changes in neural circuit properties that alter behavior (Knapp and Dowling, 1987; Maguire and Werblin, 1994; Greengard, 2001). In mammalian cells, dopamine activates a second-messenger pathway that leads to the phosphorylation of the AMPA subtype of ionotropic glutamate receptors (iGluRs) (Greengard et al., 1999; Snyder et al., 2000; Green- 
gard, 2001). Phosphorylation of iGluRs results in the modification of receptor kinetics (Roche et al., 1996). This modulation may persist on the order of minutes, consistent with the time course of ARS. This process may also be involved in longterm changes in synaptic efficacy (Barria et al., 1997).

To understand the mechanisms controlling ARS and the possible roles of dopaminergic and glutamatergic signaling, we studied this behavior in the nematode Caenorhabditis elegans. In response to attractive stimuli, such as food, C. elegans modulates its turning frequency and navigates up a gradient of chemical cues (Croll, 1975; Pierce-Shimomura et al., 1999). Dopaminergic signaling mediates the locomotory response to food (Sawin et al., 2000) and also plays a role in feeding and egg laying (Schafer and Kenyon, 1995; Weinshenker et al., 1995). Four pairs of bilaterally symmetrical dopaminergic sensory neurons have been identified in the C. elegans hermaphrodite (Sulston et al., 1975). The command interneurons of the locomotory control circuit express iGluRs and receive synaptic input from the dopaminergic neurons (White et al., 1986; Hart et al., 1995; Maricq et al., 1995; Brockie et al., 2001a). Mutations in iGluR subunits that are expressed in these interneurons lead to predictable changes in the frequency of high-angled turns (Zheng et al., 1999; Brockie et al., 2001b).

Here, we describe the ARS phenotype in C. elegans and show that a specific neural pathway involving dopamine and glutamate mediates this behavior. Well-fed worms show stereotypical ARS behavior and, thus, turn more frequently immediately after removal from food compared with $30 \mathrm{~min}$ of food deprivation. This locomotion response to food deprivation can be reset by brief exposures to food, consistent with the ecological descriptions of ARS (Kareiva and Odell, 1987; Bell, 1991). Exposure to exogenous dopamine increases turning frequency, whereas a dopamine antagonist, raclopride, is sufficient to eliminate ARS. Ablation of the dopaminergic neurons also eliminates ARS but is rescued by preincubation on dopamine. Downstream of the dopaminergic neurons, we show that mutations in genes required for glutamatergic signaling are sufficient to eliminate ARS. Thus, dopaminergic modulation of turning frequency requires functional glutamatergic signaling.

\section{Materials and Methods}

Strains and DNA constructions. Nematodes were raised at $20^{\circ} \mathrm{C}$ under standard laboratory conditions on agar plates covered in the Escherichia coli strain HB101 (Brenner, 1974; Sawin et al., 2000). Wild-type nematodes were $C$. elegans strain N2. The mutant strains used were ced1(e1735), glr-1(ky176), glr-2(ak10), eat-4(ky5), glr-2(ak10) glr-1(ky176), and cat-2(e1112). Rescue transgenic lines were generated for $g l r-1$ (ky176) and $g l r-2$ (ak10) by injecting $n m r-1:: G F P(\mathrm{pPB} 1 ; 40 \mu \mathrm{g} / \mathrm{ml})$ with either a $g l r-1$ (pV1) or $g l r-2$ (pPB57) genomic clone $(60 \mu \mathrm{g} / \mathrm{ml})$ (Maricq et al., 1995; Mellem et al., 2002). The transgenic dat-1::ICE strain was generated by injecting lin-15(n765ts) mutants with a lin-15 rescuing plasmid (pJM23; $30 \mu \mathrm{g} / \mathrm{ml}$ ), along with dat-1::GFP (pTH6; $65 \mu \mathrm{g} / \mathrm{ml}$ ) and dat-1::ICE (pTH5; $65 \mu \mathrm{g} / \mathrm{ml}$ ). Cell death was confirmed by observing apoptotic bodies in transgenic dat-1::GFP; dat-1::ICE; ced-1(e1735) mutants (Zheng et al., 1999). The dat-1::GFP; dat-1::ICE transgenic worms used for the experiments were first identified by green fluorescent protein (GFP) expression in L1 larval and then by the absence of GFP on the day of the experiment.

$A R S$ and recovery assay. Young adult worms were removed from the culture plate using a glass pick and allowed to move freely on a food-free agar surface to remove residual food. Worms were then moved to an unseeded (no food) observation plate ( $5 \mathrm{~cm}$ diameter) containing $5 \mathrm{ml}$ of media $\left(1 \mu \mathrm{MgSO}_{4}, 1 \mu \mathrm{M} \mathrm{CaCl}_{2}, 5 \mu \mathrm{MPO}_{4}, \mathrm{pH} 6\right.$, and $2 \%$ Difco-Bacto agar) prepared the day before and kept at $20^{\circ} \mathrm{C}$. The observation plate was placed under a DCR-TRV 900 digital video camera (Sony, Tokyo, Japan) and a ring light was used to provide even illumination of the agar surface. Frames were captured at a rate of one per second. For the ARS assay, worms were observed during the first $5 \mathrm{~min}$ and again after a total of 30 min had elapsed (last observation period). For the recovery assay (Fig. 1) worms were moved to a seeded plate $60 \mathrm{sec}$ before the last observation period. Worms that were not exposed to food were placed on the agar surface beside the bacterial lawn for $60 \mathrm{sec}$. Worms that were exposed to food were first transferred to the bacterial lawn for either 2 or $50 \mathrm{sec}$ and then moved to the agar surface beside the bacteria for 58 or $10 \mathrm{sec}$, respectively. Using the Dynamic Image Analysis System (version 3.2; Solltech, Oakdale, IA), we reconstructed the paths of individual worms and analyzed the turning angle at $1 \mathrm{sec}$ intervals. These data were then filtered using a program written in Python, which identified high-angled turns based on the description by Pierce-Shimomura et al. (1999). The data for each worm was then averaged. Worms that stopped moving during the assay (velocity, $<0.05 \mathrm{~mm} / \mathrm{sec}$ ) or were otherwise visibly paralyzed were removed from the data set. These worms represented $<1 \%$ of all path data. The data were averaged for all worms on a given plate such that plates (not individual worms) represent each data point. Statistical comparisons for different strains in the ARS assay used repeatedmeasures ANOVA with strain and observation time (repeated factor) as the nominal variables. Data were considered statistically significant when the $p$ value for the interaction effect between strain and time was $<0.05$. Results for individual strains were tested by comparing early and late turning frequency using a Student's unpaired $t$ test. Results were considered significant when the $p$ value was $<0.05$.

Neurotransmitter and drug treatment. For all pharmacological experiments, except those in Figure 3, $I$ and $J$, dopamine $(0.1,1.0$, or $10 \mathrm{~mm}$ ) or raclopride (1 $\mathrm{mm}$ ) was dissolved into the agar (without salts) (Schafer and Kenyon, 1995) and allowed to equilibrate overnight at $20^{\circ} \mathrm{C}$. With the exception of Figure 4, worms were video tracked on the assay plates immediately after removal from food. For Figure 4, worms were first preincubated on unseeded plates containing either raclopride or no drug for $5 \mathrm{~min}$ before observation on plates containing no drug, dopamine, or dopamine and raclopride. Drug treatments were considered significant if, in an ANOVA, the interaction effect between strain and pharmacological treatment was statistically different $(p<0.05)$. Strains were also tested against themselves for no drug versus drug using a Student's $t$ test. For Figure 3, $I$ and $J, 300 \mu l$ of either $10 \mathrm{~mm}$ dopamine (dissolved $\mathrm{H}_{2} \mathrm{O}$ ) or $\mathrm{H}_{2} \mathrm{O}$ was applied to the surface of a bacterial lawn and allowed to dry at room temperature for $2 \mathrm{hr}$. Worms were preincubated on these plates for 5 min before the ARS assay. The cat-2 rescue experiment was performed by observing worms on food-free plates that contained $10 \mathrm{~mm}$ dopamine. Three hundred microliters of either water or dopamine were added to salt-free plates $\sim 2 \mathrm{hr}$ before the experiment. Pretreatment was considered to rescue the ARS phenotype if the interaction effect between the treated mutants and the untreated wild-type worms was not significantly different $(p>0.05)$ by repeated-measures ANOVA.

Laser ablations. Laser ablations were performed as described previously (Bargmann and Horvitz, 1991; Sawin et al., 2000). A laser microbeam was used to ablate the dopaminergic neurons during the second larval stage in transgenic worms that expressed dat-1::GFP. The dopaminergic neurons were identified as those that expressed GFP. Successful ablations were confirmed by the loss of GFP expression in adult worms. Mock-ablated and laser-operated animals were anesthetized in parallel in $10 \mathrm{~mm}$ sodium azide. Animals were allowed $2 \mathrm{~d}$ to recover, after which behavioral assays were performed on young adults.

\section{Results}

\section{C. elegans exhibits ARS behavior}

We designed an assay to measure ARS behavior in C. elegans. Worms raised on agar plates covered with food (E. coli) were transferred to an agar plate without food (transition plate) and allowed to move approximately two body lengths to remove residual bacteria. Worms were then transferred to a second foodfree agar plate (observation plate), where they moved undisturbed for $35 \mathrm{~min}$ (Fig. $1 \mathrm{~A}$ ). The first and last $5 \mathrm{~min}$ of this period were recorded on digital video and analyzed for the frequency of 
A

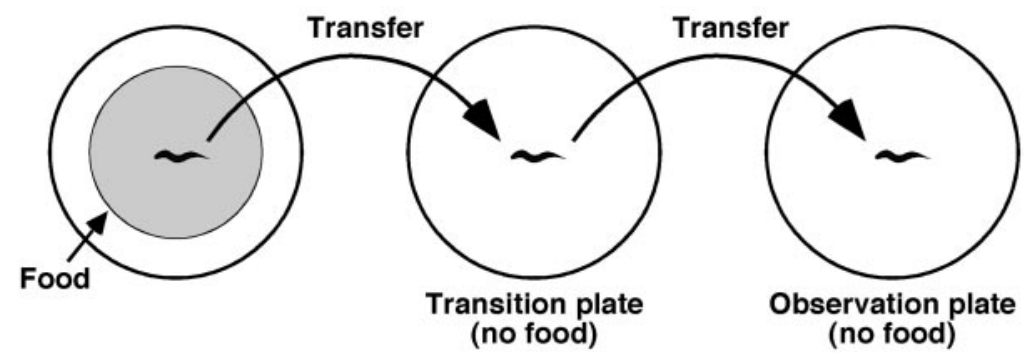

B

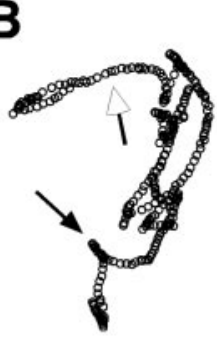

0-5 minutes

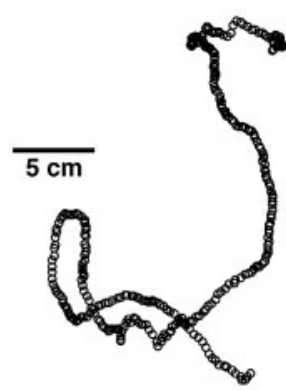

30-35 minutes

\section{C}

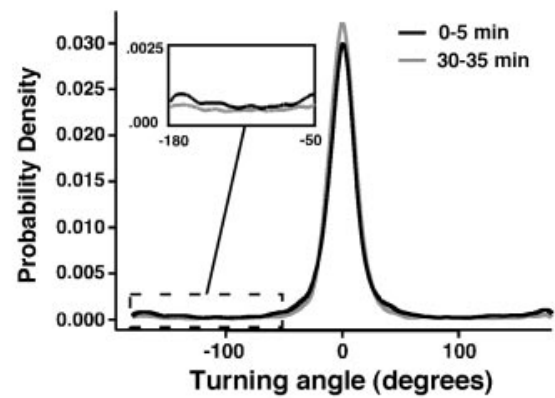

D

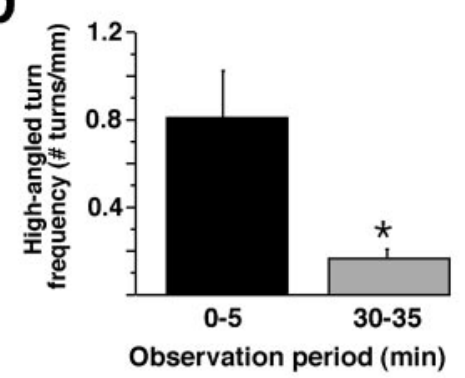

E

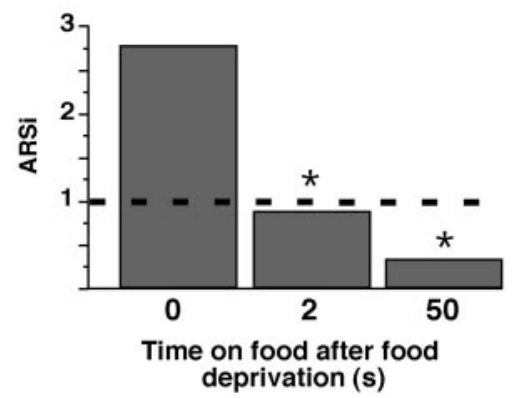

Figure 1. C. elegans does an ARS in response to food deprivation. A, The ARS assay. Worms were transferred from a plate seeded with food to a transition plate (no food) where any residual food attached to the worm was removed. The worm was then transferred to the observation plate (no food) where the turn frequency was recorded. $B$, A wild-type worm path for the first $5 \mathrm{~min}$ (left) of food deprivation. Each circle represents the position of the center of the worm at 1 sec intervals. The single arrow shows a high-angled turn. The open arrow shows a region of "running." $\mathrm{A} 5 \mathrm{~min}$ worm path after $30 \mathrm{~min}$ (right) of food deprivation shows a complete absence of high-angled turns. C, The distribution of turning angles on a food-free agar plate. Positive and negative values represent the amplitude of the turn to the right or left, respectively. The black line represents 6000 turns (cumulative from 20 worms) during the first observation period. The gray line represents 6000 turns during the last observation period. The distributions are significantly different by the Kolmogorov-Smirnov test $(p<0.05)$. The boxed inset shows the region of interest for high-angled turns, in absolute value between 50 and $180^{\circ}$. $D$, The frequency of high-angled turns per millimeter for the first $(0-5 \mathrm{~min})$ and last ( $30-35 \mathrm{~min}$ ) observation periods $(n=10 ; n$ refers to plates unless otherwise stated). $E$, The ARSi, representing the ratio of mean turns for the first and last observation periods, after 0,2 , or 50 sec of food exposure after 29 min of food deprivation $(n=8)$. Worms exposed to food just before the second observation period show significantly more turns than worms not exposed to food. * Statistical difference $(p<0.05)$.

high-angled turns and velocity (see Materials and Methods). Typical paths for the first and last observation periods showed a gradual trend toward less frequent turning as the time since removal from food increased (Fig. 1B). A smoothed histogram of 6000 turns calculated at $1 \mathrm{sec}$ intervals (Fig. $1 C$ ) shows that the distribution of turns was bimodal and consisted primarily of turning near the forward direction (referred to as "running" by Pierce-Shimomura et al., 1999). A smaller peak near $\pm 180^{\circ}$ represents the contribution to turning made by high-angled turns, which consist of stopping and reversing direction called "reversals" and sharp head to tail turns called "omega turns" (Croll, 1975; Pierce-Shimomura et al., 1999).

The focus of our research was the effect of food deprivation on the modulation of high-angled turns, specifically those turns greater in absolute magnitude than $50^{\circ}$ (as described by Pierce-Shimomura et al., 1999). The highangled turning frequency for $5 \mathrm{~min}$ was divided by the distance traveled during that period to compare the frequency of turning for worms moving at different velocities (see Materials and Methods). This determined the frequency of turns per unit distance.

Worms exhibited an increased turning frequency immediately after removal from food relative to their turning frequency observed 30 min later (Fig. 1D). The ARS index (ARSi) describes the ratio of turns between the first and last observation period (Fig. $1 E$ ). Because a high ratio of turns for the period immediately after food exposure $(0-5 \mathrm{~min})$ to turns after a period of food deprivation ( $30-35 \mathrm{~min}$ ) is indicative of ARS, in subsequent figures we used the ARSi in addition to the raw data to better reveal genetic or pharmacological manipulations that modify the ARS phenotype (see Materials and Methods for an explanation of the statistics used to detect differences in ARSi).

Worms did not become uncoordinated, nor did they significantly change their velocity over the $35 \mathrm{~min}$ observation period in the absence of food (data not shown). This suggests that ARS results from an independent modulation of turning rather than a consequence of physiological exhaustion resulting from food deprivation.

\section{ARS behavior is reset by food but not by touch}

C. elegans changes locomotion in response to a wide variety of sensory stimuli, including vibration (Rankin et al., 1990; Wicks and Rankin, 1997), temperature (Mori and Ohshima, 1995), odorants (Ward, 1973; Bargmann et al., 1993; PierceShimomura et al., 1999), touch (Chalfie et al., 1985), and food (Sawin et al., 2000). To verify that the ARS behavior we described was in response to food and not other sensory stimuli, such as touch, we quantified the locomotory response of wild-type worms to various treatments after $29 \mathrm{~min}$ of food deprivation. After the starvation period, worms were transferred to an agar plate seeded with E. coli and allowed to move briefly through the bacterial lawn for 0,2 , or $50 \mathrm{sec}$. They were then moved to a food-free area of the agar surface adjacent to the bacterial lawn, such that the total time on the treatment plate always equaled $60 \mathrm{sec}$. Turning frequency was quantified for 5 min immediately after the treatment. These values were then compared with those observed during the first $5 \mathrm{~min}$ of food deprivation.

Worms that did not spend any time in the bacterial lawn showed a reduced frequency of high-angled turns during the last 
5 min observation period compared with that observed during the first $5 \mathrm{~min}$ of food deprivation (Fig. $1 E$ ). Thus, movement by glass pick (touch stimuli) and foraging in the proximity of food was not sufficient to reset ARS behavior. Only physical contact with $E$. coli restored turning frequency to a value equal to or above its initial frequency, thus reestablishing the ARS behavior (Fig. $1 E)$. This suggests that chemosensation alone is not sufficient to reset ARS behavior (Sawin et al., 2000). A 2 sec exposure to food was sufficient to eliminate the decrement in turning frequency. Fifty-second periods on food after starvation further increased turning, consistent with previous reports that starvation increases the sensitivity to food (Avery and Horvitz, 1990; Sawin et al., 2000).

\section{ARS behavior requires functional glutamatergic signaling} A small set of interneurons has been shown to mediate the withdrawal response to tactile stimuli (Chalfie et al., 1985) and to regulate the rate of high-angled turns (Zheng et al., 1999). These neurons express a subset of the known iGluR subunits in C. elegans, including the non-NMDA subunits GLR-1 and GLR-2, that interact to form functional heteromeric channels (Mellem et al., 2002). Mutations in the $g l r-1$ gene modulate the frequency of reversals but do not lead to uncoordinated behavior or an inability to undergo high-angled turns (Maricq et al., 1995; Mellem et al., 2002). This evidence suggests that glutamatergic signaling may regulate ARS by modulating the frequency of high-angled turns to increase turning when in preferred locations (presence of food) and reduce turning when in other locations (absence of food).

To test the hypothesis that glutamatergic signaling is required for the dynamic changes in turning during ARS, we quantified the turning frequency of $g l r-1, g l r-2$, and eat-4 mutants using the ARS assay. eat- 4 encodes the C. elegans ortholog of the mammalian glutamate vesicular transporter and is expressed in sensory neurons presynaptic to interneurons that express GLR-1 and GLR-2 (Lee et al., 1999; Bellocchio et al., 2000; Takamori et al., 2000).

We found that $g l r-1(k y 176), g l r-2(a k 10)$, and eat-4(ky5) mutants were defective in ARS, because the frequency of high-angled turns did not decrease in response to food deprivation (Fig. 2). These mutants were all capable of undergoing spontaneous highangled turns as well as reversals in response to tactile stimuli (Maricq et al., 1995; Mellem et al., 2002). Interestingly, these three mutants performed similarly in the ARS assay. The defects in ARS were rescued in transgenic $g l r-1$ and $g l r-2$ mutants that expressed a wild-type $g l r-1$ or $g l r-2$ genomic clone, respectively (Fig. 2A,B). These data suggest that glutamatergic signaling is required for ARS behavior.

\section{Dopaminergic signaling is required for ARS}

Dopamine plays a significant role in C. elegans behaviors, including locomotion, egg laying, and the basal slowing response to food (Schafer and Kenyon, 1995; Weinshenker et al., 1995; Sawin et al., 2000). The C. elegans hermaphrodite has four bilaterally symmetrical pairs of dopaminergic neurons (ADE neurons, PDE neurons, and two pairs of CEP neurons) that appear to play a role in mechanosensation (Sulston et al., 1975; Sawin et al., 2000). Ablation of these neurons using a laser microbeam is sufficient to reduce the basal slowing response to food (Sawin et al., 2000) and may also affect ARS behavior.

To investigate the role of dopaminergic signaling in ARS, we ablated the dopaminergic neurons using the human caspase interleukin-1 $\beta$-converting enzyme (ICE) (Cerretti et al., 1992;
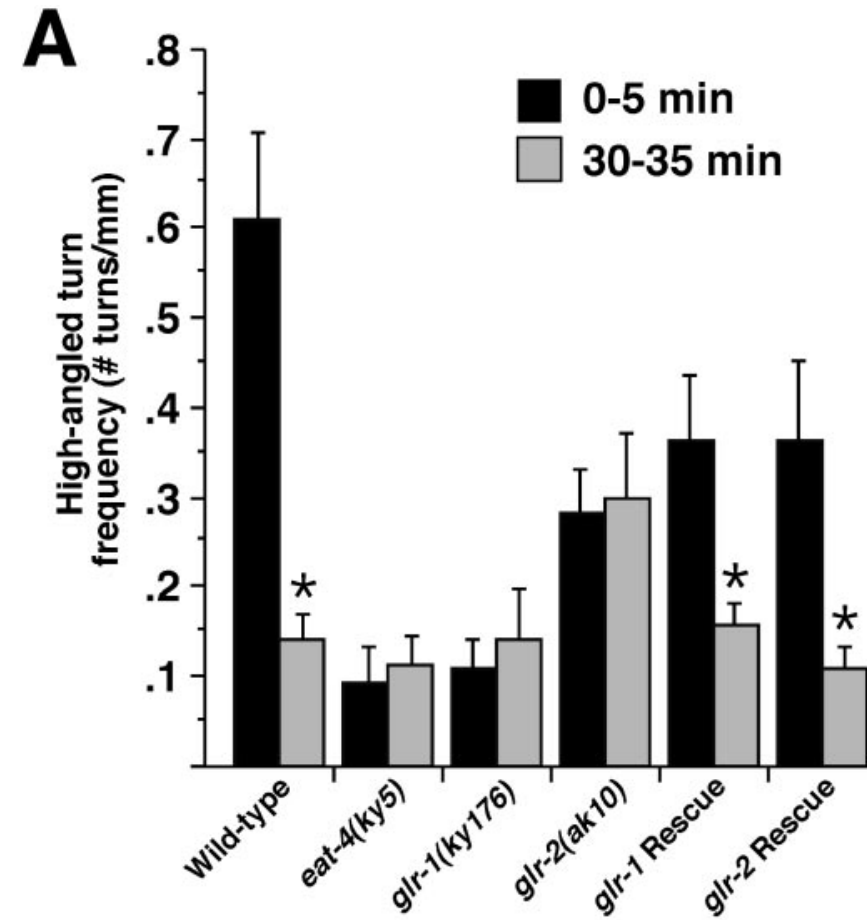

B

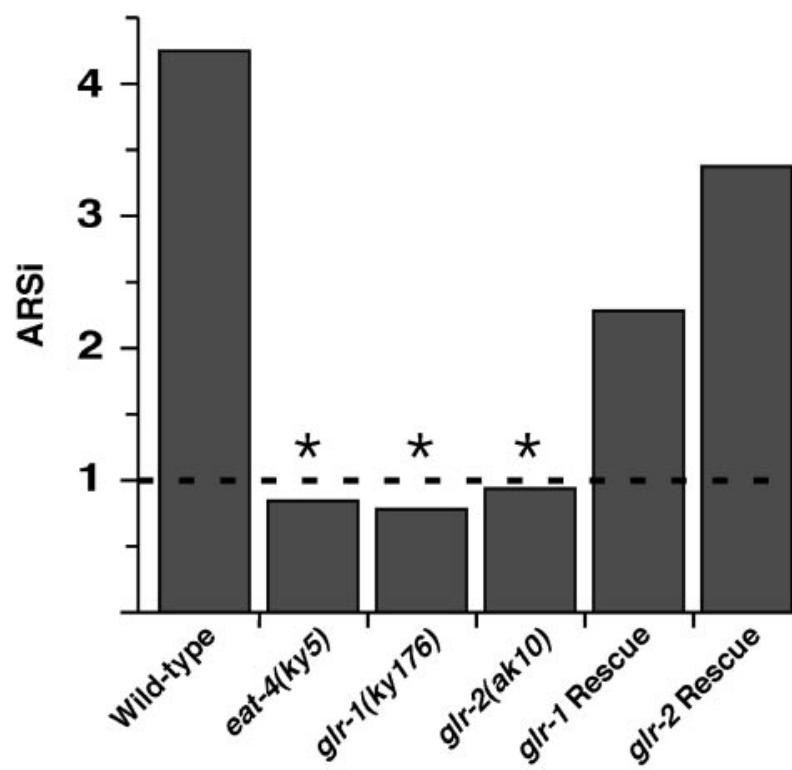

Figure 2. Defects in glutamatergic signaling alter ARS. $A$, The mean number of high-angled turns per millimeter during the first (black) and last (gray) 5-min observation period for wildtype worms; eat-4(ky5), glr-1(ky176), and glr-2(ak10) mutants; and transgenic glr-1 ( glr-1 Rescue) and glr-2 ( glr-2 Rescue) mutants that expressed an introduced wild-type glr-1 or glr-2 transgene, respectively $(n=10)$. $B$, The ARSi. *Statistical difference from self in $A$ by Student's $t$ test or from wild-type in $B$ by repeated-measures ANOVA $(p<0.05)$.

Thornberry et al., 1992). C. elegans neurons that express ICE have been shown to undergo premature cell death (Zheng et al., 1999). ICE and the reporter molecule GFP were expressed in the dopaminergic neurons of transgenic worms using upstream regulatory sequences of the dat-1 gene (Jayanthi et al., 1998). Cell death was assessed by monitoring the loss of GFP fluorescence (Fig. $3 A, B)$ and by the characteristic morphological features of apoptotic cell death (Fig. 3C,D). Transgenic worms in which all neurons were dead by GFP expression criteria failed to exhibit ARS 

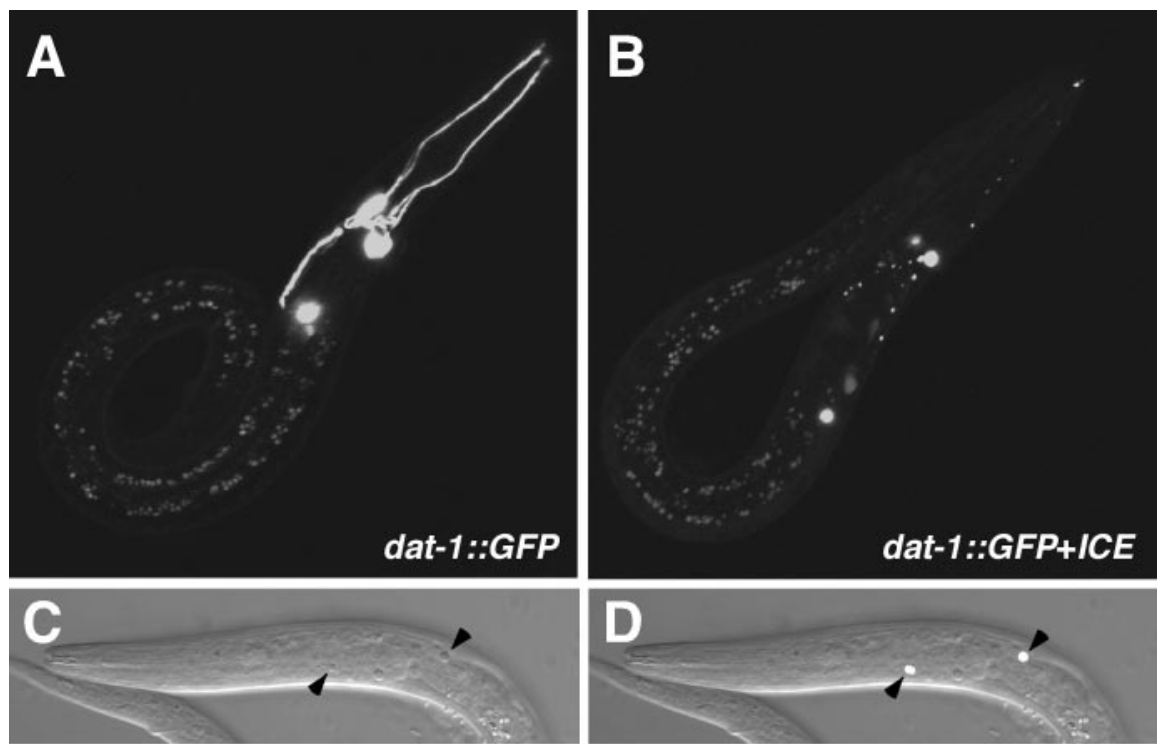

E

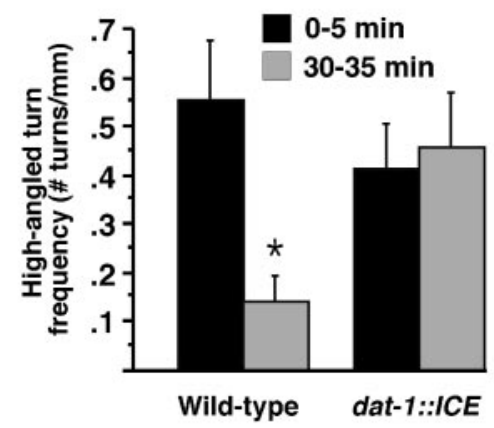

G

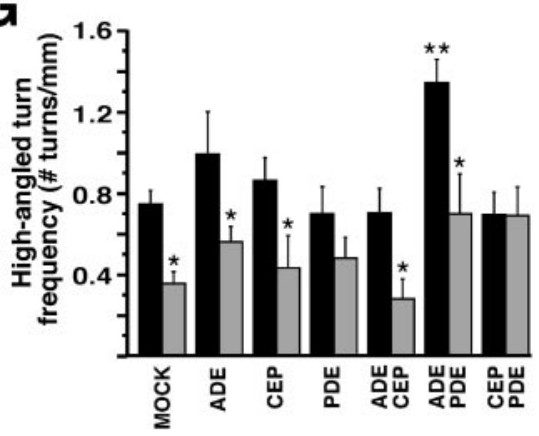

I

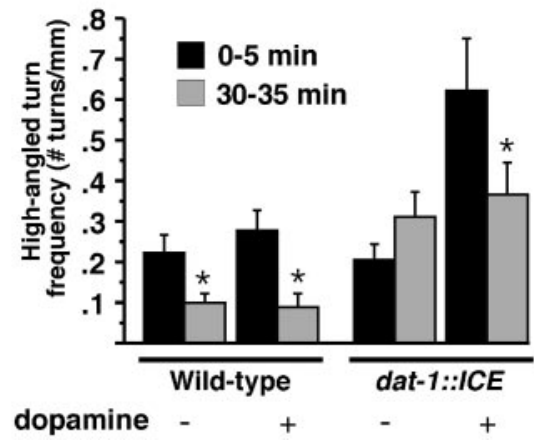

F

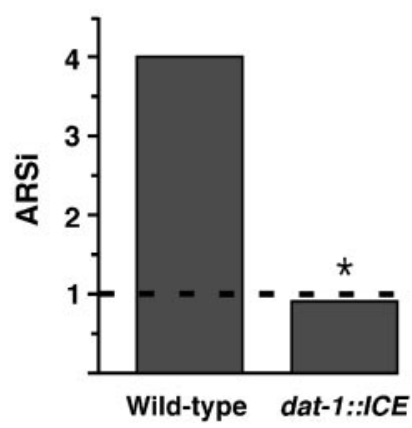

H

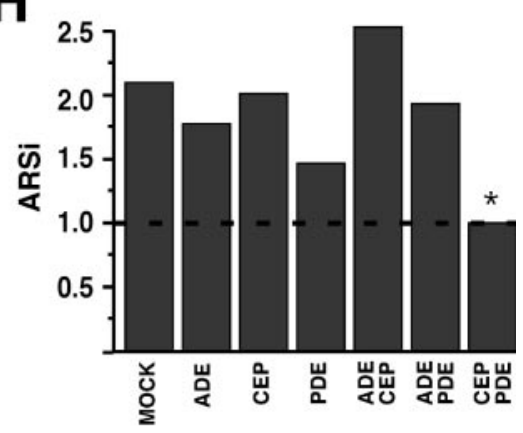

J

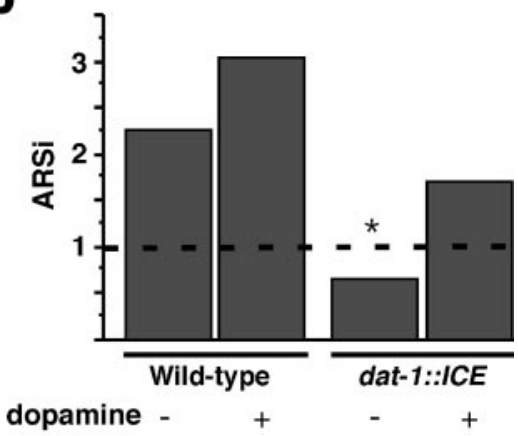

Figure 3. Dopaminergic signaling is required for ARS. A, dat-1::GFP expression in a wild-type transgenic worm. $B$, dat-1::GFP expression in a transgenic worm that also expressed dat-1::/CE. C, $D$, Apoptotic bodies (arrowheads) in transgenic ced-1 mutants that expressed both dat-1::GFP and dat-1::ICE. Nomarski ( $C$ ) and Nomarski plus GFP fluorescence (D). E, G, $I$, The frequency of high-angled turns for the first (black) and last (gray) observation periods in wild-type worms $(n=10)$ and transgenic worms that expressed dat-1::/CE $(n=10)(E)$; in mock-ablated transgenic dat-1::GFP worms ( $n=20)$ and laser-operated transgenic worms in which either the ADE $(n=6), \operatorname{CEP}(n=8), \operatorname{PDE}(n=9), \operatorname{ADE}$ and CEP $(n=5), \operatorname{ADE}$ and PDE $(n=5)$, or CEP and PDE $(n=7)$ neurons were ablated ( $G)$; and in wild-type worms $(n=10)$ and transgenic dat-1::/CE worms $(n=10)$ in either the presence or absence of exogenous 10 mm dopamine $(I)$. $F, H, J$, The ARSi for turns for worms shown in $E(F), G(H)$, and $I(J)$. ${ }^{\circ}$ Statistical difference from self $(E, G, I)$ by Student's $t$ test or from wild-type $(F, H, J)$ by repeated-measures ANOVA $(p<0.05)$. ${ }^{* *}$ Statistical difference from wild-type by Student's $t$ test $(G)$. 
after removal from food (Fig. $3 E, F$ ). This result suggests that dopaminergic neurons release dopamine in the presence of food to modulate turning frequency. We also noted that the baseline frequency of high-angled turns increased in the transgenic worms during the last observation period. This may reflect developmental compensation for the absence of dopaminergic inputs. Alternatively, dopaminergic neurons may have multiple functions such that they regulate both the baseline frequency of high-angle turns as well as ARS.

To further address this issue, we ablated specific subsets of the dopaminergic neurons using a laser microbeam. Compared with mock-ablated worms, ablation of the ADE, CEP, or PDE neurons alone had no effect on either the baseline frequency of highangled turns or on ARS behavior (Fig. 3G,H). This suggests that the neuronal regulation of high-angled turns is distributed among the dopaminergic neurons. To address this further, we ablated pairs of neurons in all combinations. Ablating both $\mathrm{ADE}$ and CEP or ADE and PDE did not eliminate ARS behavior. Interestingly, worms in which both CEP and PDE neurons were ablated no longer performed ARS, thus localizing the modulation of high-angled turns to these neurons. Furthermore, ablation of both the ADE and PDE neurons increased the baseline frequency of high-angled turns, suggesting that specific dopaminergic neurons may have distinct roles in regulating ARS behavior.

We also determined whether exogenous dopamine could rescue the ARS defects of transgenic dat-1::ICE worms. Both wildtype and transgenic worms were incubated on agar plates containing both bacteria and dopamine for $5 \mathrm{~min}$, and their ARS behavior was observed on food-free plates without dopamine. Interestingly, dat-1::ICE worms preincubated on dopamine exhibited complete rescue of the ARS response (Fig. $3 I, J$ ).

\section{Exogenous dopamine increases the frequency of high-angled turns}

Our analysis suggests that dopamine plays a role in the modulation of turning frequency. Therefore, we determined the effect of exogenously applied dopamine on turning behavior in wild-type worms. Increasing concentrations of dopamine increased the frequency of high-angled turns (Fig. 4A). To confirm that these were directly mediated by dopamine receptors, we preincubated worms for $5 \mathrm{~min}$ on agar containing the dopamine antagonist raclopride and then observed worms for 5 min after they were transferred to agar plates containing both raclopride and dopamine but not food. Raclopride blocked the effects of dopamine on turning frequency (Fig. $4 \mathrm{~B}$ ) but did not disrupt the ability of these worms to undergo high-angled turns. These data support the hypothesis that exogenous dopamine acts via dopamine receptors to increase the frequency of high-angled turns. We also tested the effect of raclopride on ARS. Exposure to raclopride after removal from food was sufficient to completely eliminate ARS (Fig. 4C,D) demonstrating that the dynamic modulation of turning requires functional dopaminergic signaling pathways.

These results demonstrate the importance of the neurotransmitter dopamine in ARS behavior. If functional dopaminergic pathways are required for ARS, we would hypothesize that cat-2 mutants, which are defective for dopamine synthesis because of a mutation in tyrosine hydroxylase (Sulston et al., 1975; Lints and Emmons, 1999), would also show defects in ARS. Consistent with our hypothesis, cat-2(e1112) mutants no longer showed ARS behavior (Fig. 5). These defects were rescued in cat-2 mutants that were observed on dopamine plates (Fig. 5). Previous work by Sawin et al. (2000) suggests that other mechanisms that control foraging might come into effect after $30 \mathrm{~min}$ of food deprivation.

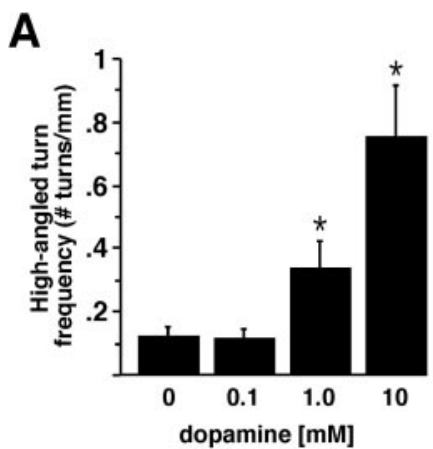

B
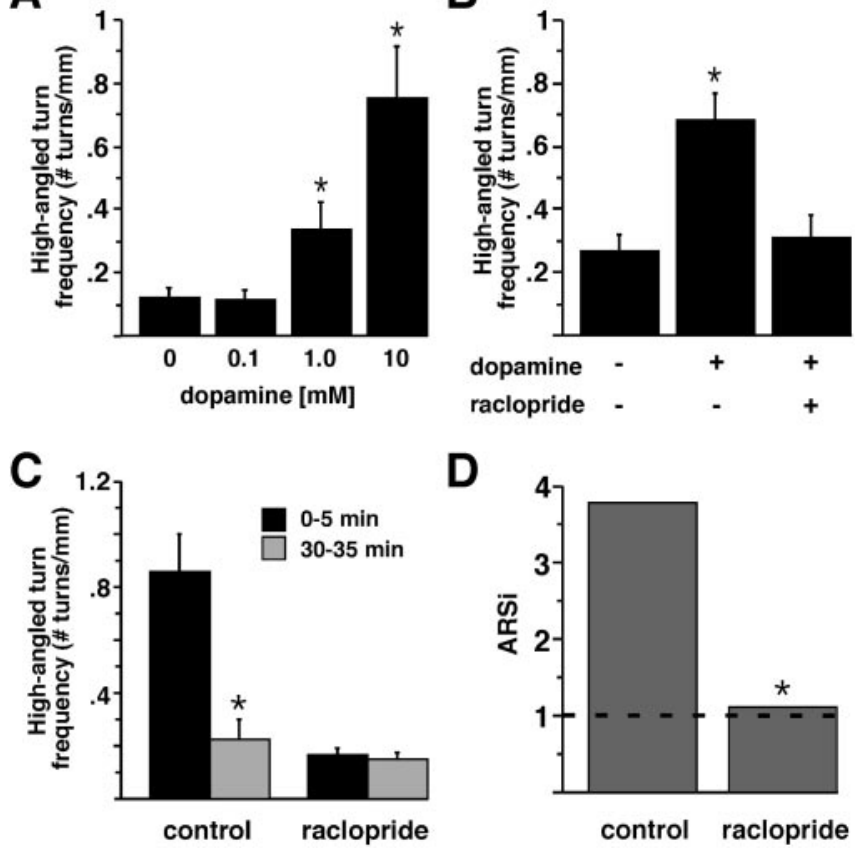

D

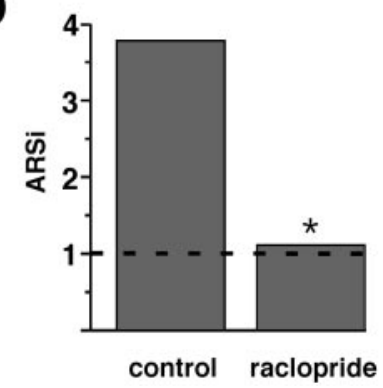

Figure 4. The dopamine antagonist raclopride eliminates ARS. A, Frequency of high-angled turns for wild-type worms in the presence of various concentrations of exogenous dopamine. Concentrations higher than $10 \mathrm{~mm}$ led to paralysis during the observation period. No visibly paralyzed worms were included in the analysis. $B$, The frequency of high-angled turns in wildtype worms in either the presence or absence of exogenous dopamine and raclopride $(n=8)$. $C$, The frequency of high-angled turns for the first (black) and last (gray) observation periods for wild-type worms in either the presence or absence of exogenous raclopride $(n=10)$. $D$, The ARSi shows a significant reduction in the turning ratio between the first and last observation periods. *Statistical difference from self $(C)$ by Student's $t$ test or from wild-type $(D)$ by repeated-measures ANOVA $(p<0.05)$
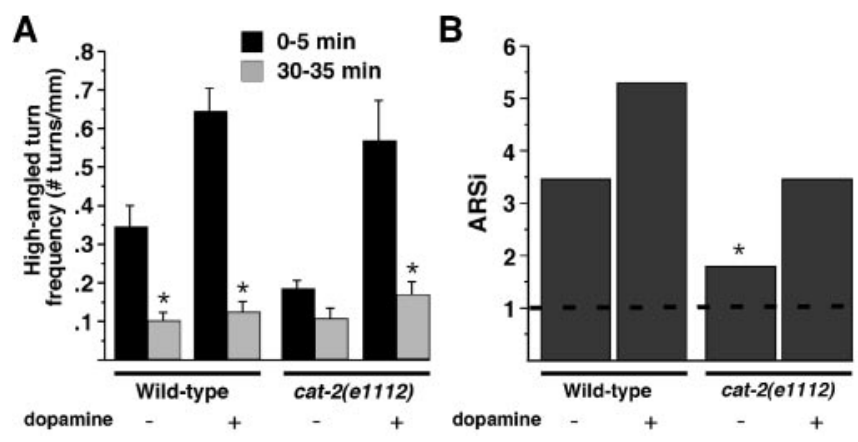

Figure 5. cat-2 mutants are defective in ARS. $A$, The frequency of high-angled turns during the first and last 5 min observation periods for wild-type worms and cat-2(e1112) mutants in either the presence or absence of exogenous dopamine. $B$, The ARSi. *Statistical difference from self $(A)$ by Student's $t$ test or from wild-type or cat-2(e1112) plus dopamine $(B)$ by repeatedmeasures ANOVA $(p<0.05)$.

Figure 5 shows that wild-type worms and cat-2 mutants adapt to dopamine over a $30 \mathrm{~min}$ period (Schafer and Kenyon, 1995), further establishing the role for nondopaminergic mechanisms in response to starvation.

\section{Glutamate receptors are required for dopaminergic effects on locomotion}

Dopamine may regulate ARS by modulating glutamatergic neural circuits or via parallel pathways independent of glutamatergic signaling. To test the hypothesis that dopamine modifies glutamatergic signaling, we applied exogenous dopamine to eat-4, 
A

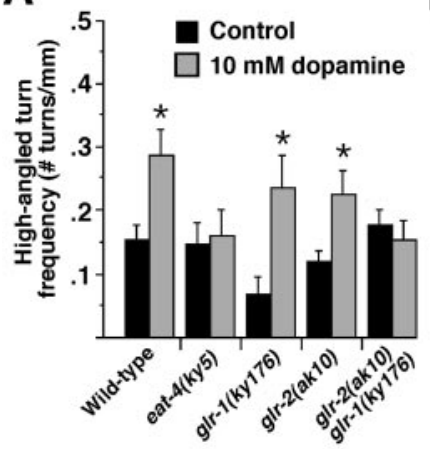

B

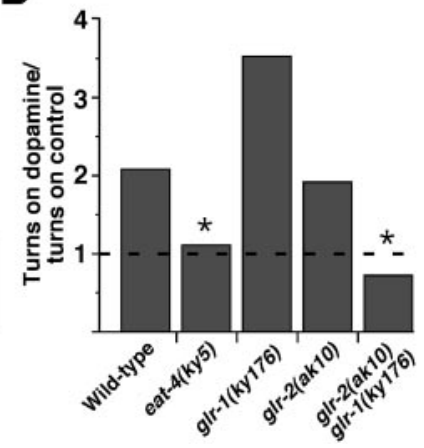

Figure 6. Dopamine fails to increase turning in worms defective in glutamatergic signaling. $A$, The frequency of high-angled turns for worms observed on plates with $10 \mathrm{~mm}$ exogenous dopamine (gray) or on control plates without dopamine (black) $(n=10) . B$, The ratio of turns in the presence of dopamine to turns on control plates. *Statistical difference from self $(A)$ by Student's $t$ test or from wild-type $(B)$ by ANOVA $(p<0.05)$.

$g l r-1$, and $g l r-2$ mutants and to the $g l r-2 g l r-1$ double mutant. Worms were moved to an observation plate in either the presence (test plate) or absence (control plate) of $10 \mathrm{~mm}$ dopamine, and their turns were measured for 5 min. The eat-4(ky5) mutants and the $g l r-2 g l r-1$ double mutants did not modulate their frequency of high-angled turns in the presence of dopamine compared with wild-type (Fig. 6). This suggests that dopamine is acting via glutamatergic signaling pathways to modulate the frequency of high-angled turns.

Interestingly, worms with a mutation in either $g l r-1$ or $g l r-2$ alone exhibited a response to dopamine (Fig. 6), suggesting that other iGluR subunits may interact with either GLR-1 or GLR-2 to form functional channels that mediate the turning response to dopamine. Because $g l r-1$ and $g l r-2$ single mutants fail to show ARS in response to food deprivation (Fig. 2), but still show a response to dopamine (Fig. 6), dopamine may be acting via other glutamatergic or nonglutamatergic pathways to mediate a turning response to environmental stimuli. It is also possible that GLR-1 and GLR-2 can each function as a homomeric channel, explaining the responses of the mutants to dopamine.

\section{Discussion}

We show that $C$. elegans performs an ARS in response to food deprivation that is detectable over a $35 \mathrm{~min}$ period and is dependent on dopaminergic and glutamatergic signaling. ARS behavior is observed in a wide variety of animals (Bell, 1991), including house flies (White et al., 1984), leeches (O'Gara et al., 1991), moths (Vickers, 2000), populations of ladybug beetles (Kareiva and Odell, 1987), and rodents (Benedix, 1993). Specific molecular mechanisms have not been reported for the control of these behaviors. However, there is substantial support for the dopaminergic control of feeding and search behaviors (O'Gara et al., 1991; Sawin et al., 2000; Foltin, 2001). Our results show how dopamine may regulate glutamatergic signaling in the locomotory control circuit to modulate turning in C. elegans and, therefore, generate a behavioral response to food deprivation. However, we cannot rule out that dopamine acts in a pathway that is parallel to glutamatergic signaling.

\section{ARS is controlled by a dopaminergic response to food deprivation}

The ability to inhibit ARS in C. elegans with the dopamine receptor antagonist raclopride, by ablation of the dopaminergic neu- rons, or by a mutation in cat-2 that prevents dopamine synthesis suggests that the modulation of turning in response to food deprivation is under the direct control of dopamine. This hypothesis was further supported by the observation that exogenously applied dopamine restored ARS behavior in transgenic worms in which the dopaminergic neurons were ablated using the human caspase ICE.

Previous observations of the locomotion rate in C. elegans found that a slowing response to food exposure, called the basal slowing response, required functional dopaminergic signaling (Sawin et al., 2000). This result, considered together with our observations, provides a mechanism for ARS in C. elegans in which dopaminergic neurons transduce a food stimulus into a behavioral response consisting of a change in the frequency of high-angled turns.

\section{Dopamine acts via glutamatergic signaling pathways to modulate turning frequency}

Previous work (Zheng et al., 1999; Brockie et al., 2001b; Mellem et al., 2002) has revealed a strong relationship between the functional properties of iGluRs and the control of turning frequency. For example, expression of a dominantly active iGluR in the command interneurons is sufficient to increase the frequency of reversals per minute by an order of magnitude (Zheng et al., 1999). In addition, deletion of the iGluR subunits GLR-1 and NMR-1 (NMDA type) in the command interneurons results in a significant decrement in the frequency of high-angled turns (Brockie et al., 2001b; Mellem et al., 2002).

A mechanism for the modulation of iGluR kinetics by dopamine has been described in detail for vertebrates (Greengard, 2001). The mechanism involves a dopamine-activated secondmessenger pathway that leads to the phosphorylation of AMPAtype iGluRs (Greengard et al., 1999; Snyder et al., 2000; Greengard, 2001). Ultimately, this pathway results in the net activation of DARPP-32, a dopamine- and cAMP-regulated phosphoprotein that acts as a potent inhibitor of protein phosphatase (Yan et al., 1999). Activation of DARPP-32 slows the dephosphorylation of AMPA-type iGluRs, thereby modulating the kinetic properties of the receptors. The time course of the change in receptor kinetics is on the order of minutes (Greengard, 2001). A similar process may explain ARS behavior in C. elegans and, thus, provide a direction for future study of this plastic behavior.

Our work further contributes to this line of reasoning by showing that ARS behavior is dependent on iGluRs. We have shown that both dopaminergic and glutamatergic signaling are necessary for the modulation of high-angled turns after food deprivation. The command interneurons that express the GLR-1 and GLR-2 subunits receive synaptic input from the dopaminergic sensory neurons, suggesting that dopaminergic signaling may directly modify iGluRs to control the frequency of high-angled turns and, thus, ARS behavior.

Interestingly, $g l r-1$ and $g l r-2$ mutants (Maricq et al., 1995; Mellem et al., 2002), and worms with mutations in the EAT-4 glutamate vesicular transporter (Lee et al., 1999), do not have coordination defects, nor do they show a reduction in the velocity of movement. Furthermore, these mutants can still perform high-angled turns in response to certain external stimuli. Compared with $g l r-1$ mutants, $g l r-2$ mutants have less severe defects in behaviors regulated by glutamate (Mellem et al., 2002). We also found that although $g l r-2$ mutants have a high level of baseline high-angled turns, they are, nevertheless, deficient in ARS. These data show that eliminating ARS behavior in iGluR mutants is not simply caused by a reduced frequency of high-angled turns dur- 
A

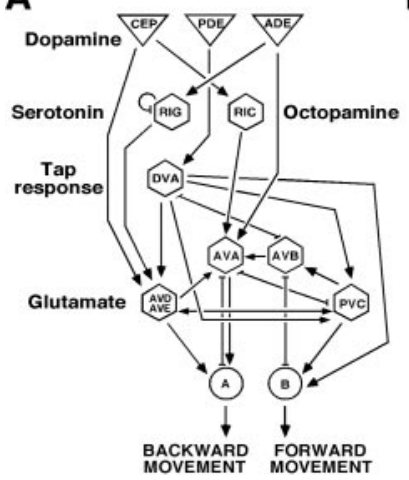

B

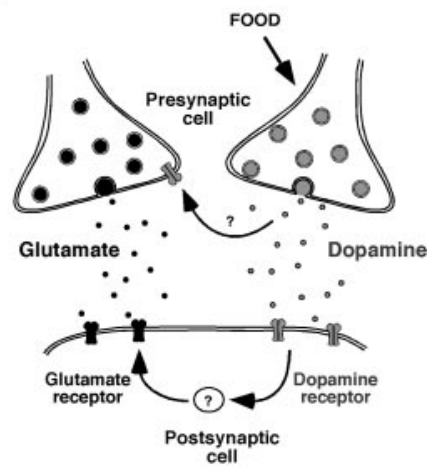

Figure 7. A model that describes the control of ARS. A, A diagrammatic representation, reconstructed from White et al. (1986), of the neural circuit proposed to control ARS that shows the position of the dopaminergic sensory neurons (CEP, PDE, and ADE) relative to the command interneurons that express GLR-1 and GLR-2 (AVA, AVB, AVD, AVE, and PVC). Postsynaptic interneurons that express serotonin (RIG), octopamine (RIC), and those involved in the tap response (DVA) (Rankin et al., 1990) are also shown. Sensory neurons (triangles), interneurons (hexagons), and motor neurons (circles) are shown. The $\rightarrow$ and $\mid$ represent chemical synapses and gap junctions, respectively. $B$, We propose a model in which food activates dopaminergic sensory neurons, causing them to release dopamine onto downstream neurons. This may lead to the modulation of glutamate receptors in the postsynaptic cell via an unidentified intermediate, or to increased glutamate release from presynaptic glutamatergic neurons. The activation of glutamate receptors results in an increased frequency of high-angled turns.

ing the first observation period. Taken together, our data suggest that glutamatergic signaling is required for the modulation of turning frequency in response to specific environmental stimuli and, thus, ARS behavior. Therefore, we propose a mechanism whereby dopaminergic sensory neurons; activated by a change in food stimuli, release dopamine to modulate downstream glutamatergic signaling (Fig. 7). Dopamine may directly modulate glutamate release or uptake, the properties of iGluRs in the command interneurons, or possibly the properties of other interneurons in parallel pathways that interact with these neurons.

\section{Additional mechanisms for the modulation of turning frequency}

Given the importance of identifying resources in the environment, it is likely that additional signaling pathways transduce information about the presence or absence of food into a behavioral response. Previous work on the locomotion rate in C. elegans shows that the response to food after long periods of starvation is transduced by the neurotransmitter serotonin (Sawin et al., 2000). Other research shows a role for octopamine in starvation-induced behaviors and serotonin in feeding-induced behaviors (Horvitz et al., 1982). The lack of ARS in $g l r-1$ and $g l r-2$ mutants suggests that serotonin and octopamine might also be required to modulate turning via a circuit that is dependent on glutamatergic signaling. The existence of other pathways provides a mechanism for developmental compensation and may thereby explain the lack of complete elimination of ARS in cat-2 mutants, which appear to be deficient in dopamine synthesis (Sulston et al., 1975), as well as the change in baseline turning frequency observed in transgenic dat-1::ICE worms. The high baseline turning frequency in these transgenic worms compared with cat- 2 mutants suggests that the dopaminergic neurons, required for ARS, may have an additional function that acts to downregulate the frequency of high-angled turns. We have found that the baseline turning frequency is modulated by both dopamine and glutamate. Mutations in $g l r-1, g l r-2$, eat-4, and cat-2 and treatment with raclopride, all associated with disrupted ARS, also decrease the baseline turning frequency. However, as we have shown for $g l r-2$ and $g l r-1$ mutants, the effects on high-angled turns and ARS are partially separable. In addition, ablation of dopaminergic neurons caused a severe decrease in ARS without significantly affecting high-angled turns. We also believe that it is unlikely that the loss of ARS behavior in cat-2 mutants is attributable simply to a decreased frequency of high-angled turns during the first observation period. First, our analysis shows that cat-2 mutants are still capable of performing these turns, although at a lower frequency. Second, we do not observe the proportional decrease in turn frequency over the observation period that is characteristic of ARS behavior.

Given the range of environmental stimuli that influence turning behavior in C. elegans, we suspect that many pathways will influence ARS behavior. However, our research identifies the importance of dopaminergic and glutamatergic signaling in ARS, thus providing a framework for additional investigation of the underlying neural mechanism.

\section{References}

Andow DA, Kareiva PM, Levin SA, Okubo A (1990) Spread of invading organisms. Landscape Ecol 4:177-188.

Avery L, Horvitz HR (1990) Effects of starvation and neuroactive drugs on feeding in Caenorhabditis elegans. J Exp Zool 253:263-270.

Bargmann CI, Horvitz HR (1991) Chemosensory neurons with overlapping functions direct chemotaxis to multiple chemicals in C. elegans. Neuron 7:729-742.

Bargmann CI, Hartwieg E, Horvitz HR (1993) Odorant-selective genes and neurons mediate olfaction in C. elegans. Cell 74:515-527.

Barnes S, Syed NI, Bulloch AG, Lukowiak K (1994) Modulation of ionic currents by dopamine in an interneurone of the respiratory central pattern generator of Lymnaea stagnalis. J Exp Biol 189:37-54.

Barria A, Muller D, Derkach V, Griffith LC, Soderling TR (1997) Regulatory phosphorylation of AMPA-type glutamate receptors by CaM-KII during long-term potentiation. Science 276:2042-2045.

Bell WJ (1991) Searching behavior: the behavioral ecology of finding resources. New York: Chapman and Hall.

Bellocchio EE, Reimer RJ, Fremeau Jr RT, Edwards RH (2000) Uptake of glutamate into synaptic vesicles by an inorganic phosphate transporter. Science 289:957-960.

Benedix JH (1993) Area-restricted search by the plains pocket gopher (Geomys bursarius) in tallgrass prairie habitat. Behav Ecol 4:318-324.

Berke JD, Hyman SE (2000) Addiction, dopamine, and the molecular mechanisms of memory. Neuron 25:515-532.

Brenner S (1974) The genetics of Caenorhabditis elegans. Genetics 77:71-94.

Brockie PJ, Madsen DM, Zheng Y, Mellem J, Maricq AV (2001a) Differential expression of glutamate receptor subunits in the nervous system of $C$. elegans and their regulation by the homeodomain protein UNC-42. J Neurosci 21:1510-1522.

Brockie PJ, Mellem JE, Hills T, Madsen DM, Maricq AV (2001b) The C. elegans glutamate receptor subunit NMR-1 is required for slow NMDAactivated currents that regulate reversal frequency during locomotion. Neuron 31:617-630.

Cameron DL, Williams JT (1993) Dopamine D1 receptors facilitate transmitter release. Nature 366:344-347.

Cerretti DP, Kozlosky CJ, Mosley B, Nelson N, Ness KV, Greenstreet TA, March CJ, Kronheim SR, Druck T, Cannizzaro LA, Huebner K, Black RA (1992) Molecular cloning of the interleukin- $1 \beta$-converting enzyme. Science 256:97-100.

Chalfie M, Sulston JE, White JG, Southgate E, Thomson JN, Brenner S (1985) The neural circuit for touch sensitivity in Caenorhabditis elegans. J Neurosci 5:956-964.

Croll NA (1975) Components and patterns in the behaviour of the nematode Caenorhabditis elegans. J Zool Lond 176:159-176.

Dulawa SC, Grandy DK, Low MJ, Paulus MP, Geyer MA (1999) Dopamine D4 receptor-knock-out mice exhibit reduced exploration of novel stimuli. J Neurosci 19:9550-9556.

Faraone SV, Doyle AE, Mick E, Biederman J (2001) Meta-analysis of the 
association between the 7-repeat allele of the dopamine $\mathrm{D}(4)$ receptor gene and attention deficit hyperactivity disorder. Am J Psychiatry 158:1052-1057.

Foltin RW (2001) Effects of amphetamine, dexfenfluramine, diazepam, and other pharmacological and dietary manipulations on food "seeking" and "taking" behavior in non-human primates. Psychopharmacology (Berl) 158:28-38.

Greengard P (2001) The neurobiology of slow synaptic transmission. Science 294:1024-1030.

Greengard P, Allen PB, Nairn AC (1999) Beyond the dopamine receptor: the DARPP-32/protein phosphatase-1 cascade. Neuron 23:435-447.

Grunbaum D (1998) Using spatially explicit models to characterize foraging performance in heterogeneous landscapes. Am Naturalist 151:97-115.

Grunbaum D (2000) Advection-diffusion equations for internal statemediated random walks. SIAM J Appl Math 61:43-73.

Harris-Warrick RM, Coniglio LM, Barazangi N, Guckenheimer J, Gueron S (1995) Dopamine modulation of transient potassium current evokes phase shifts in a central pattern generator network. J Neurosci 15:342-358.

Hart AC, Sims S, Kaplan JM (1995) Synaptic code for sensory modalities revealed by C. elegans GLR-1 glutamate receptor. Nature 378:82-85.

Hietala J, Syvalahti E (1996) Dopamine in schizophrenia. Ann Med 28:557-561.

Hollerman JR, Schultz W (1998) Dopamine neurons report an error in the temporal prediction of reward during learning. Nat Neurosci 1:304-309.

Horvitz HR, Chalfie M, Trent C, Sulston JE, Evans PD (1982) Serotonin and octopamine in the nematode Caenorhabditis elegans. Science 216:1012-1014.

Jayanthi LD, Apparsundaram S, Malone MD, Ward E, Miller DM, Eppler M, Blakely RD (1998) The Caenorhabditis elegans gene T23G5.5 encodes an antidepressant- and cocaine-sensitive dopamine transporter. Mol Pharmacol 54:601-609.

Kareiva P, Odell G (1987) Swarms of predators exhibit "preytaxis" if individual predators use area-restricted search. Am Naturalist 130:233-270.

Keaser T, Schimda A, Motro U (1996) Innate movement rules in foraging bees: flight distances are affected by recent rewards and are correlated with choice of flower type. Behav Ecol Sociobiol 39:381-388.

Knapp AG, Dowling JE (1987) Dopamine enhances excitatory amino acidgated conductances in cultured retinal horizontal cells. Nature 325:437-439.

Lee RY, Sawin ER, Chalfie M, Horvitz HR, Avery L (1999) EAT-4, a homolog of a mammalian sodium-dependent inorganic phosphate cotransporter, is necessary for glutamatergic neurotransmission in Caenorhabditis elegans. J Neurosci 19:159-167.

Lints R, Emmons SW (1999) Patterning of dopaminergic neurotransmitter identity among Caenorhabditis elegans ray sensory neurons by a TGFbeta family signaling pathway and a Hox gene. Development 126:5819-5831.

Maguire G, Werblin F (1994) Dopamine enhances a glutamate-gated ionic current in OFF bipolar cells of the tiger salamander retina. J Neurosci 14:6094-6101.

Maricq AV, Peckol E, Driscoll M, Bargmann CI (1995) Mechanosensory signalling in C. elegans mediated by the GLR-1 glutamate receptor. Nature [Erratum (1996) 379:749] 378:78-81.

Marsden CD (1992) Dopamine and basal ganglia disorders in humans. Semin Neurosci 4:171-178.

Mellem JE, Brockie PJ, Zheng Y, Madsen DM, Maricq AV (2002) Decoding of polymodal sensory stimuli by postsynaptic glutamate receptors in $C$. elegans. Neuron 36:933-944.

Mori I, Ohshima Y (1995) Neural regulation of thermotaxis in Caenorhabditis elegans. Nature 376:344-348.

Nicola SM, Surmeier J, Malenka RC (2000) Dopaminergic modulation of neuronal excitability in the striatum and nucleus accumbens. Annu Rev Neurosci 23:185-215.

O'Gara BA, Chae H, Latham LB, Friesen WO (1991) Modification of leech behavior patterns by reserpine-induced amine depletion. J Neurosci 11:96-110

Pierce-Shimomura JT, Morse TM, Lockery SR (1999) The fundamental role of pirouettes in Caenorhabditis elegans chemotaxis. J Neurosci 19:9557-9569.
Rankin CH, Beck CD, Chiba CM (1990) Caenorhabditis elegans: a new model system for the study of learning and memory. Behav Brain Res 37:89-92.

Rocha BA, Fumagalli F, Gainetdinov RR, Jones SR, Ator R, Giros B, Miller GW, Caron MG (1998) Cocaine self-administration in dopaminetransporter knockout mice. Nat Neurosci 1:132-137.

Roche K, O’Brien R, Mammen A, Bernhardt J, Huganir R (1996) Characterization of multiple phosphorylation sites on the AMPA receptor GluR1 subunit. Neuron 16:1179-1188.

Sawin ER, Ranganathan R, Horvitz HR (2000) C. elegans locomotory rate is modulated by the environment through a dopaminergic pathway and by experience through a serotonergic pathway. Neuron 26:619-631.

Schafer WR, Kenyon CJ (1995) A calcium-channel homologue required for adaptation to dopamine and serotonin in Caenorhabditis elegans. Nature 375:73-78.

Slatkin M (1985) Gene flow in natural population. Ann Rev Ecol System 16:393-430.

Snyder GL, Allen PB, Fienberg AA, Valle CG, Huganir RL, Nairn AC, Greengard P (2000) Regulation of phosphorylation of the GluR1 AMPA receptor in the neostriatum by dopamine and psychostimulants in vivo. J Neurosci 20:4480-4488.

Sulston J, Dew M, Brenner S (1975) Dopaminergic neurons in the nematode Caenorhabditis elegans. J Comp Neurol 163:215-226.

Szczypka MS, Kwok K, Brot MD, Marck BT, Matsumoto AM, Donahue BA, Palmiter RD (2001) Dopamine production in the caudate putamen restores feeding in dopamine-deficient mice. Neuron 30:819-828.

Takamori S, Rhee JS, Rosenmund C, Jahn R (2000) Identification of a vesicular glutamate transporter that defines a glutamatergic phenotype in neurons. Nature 407:189-194.

Thornberry NA, Bull HG, Calaycay JR, Chapman KT, Howard AD, Kosturea MJ, Miller DK, Molineaux SM, Weidner JR, Aunins J, Elliston KO, Ayala JM, Casano FJ, Chin J, Ding GJ, Egger LA, Gaffney EP, Limjuco G, Palyha OC, Raju SM, et al. (1992) A novel heterodimeric cystein protease is required for interleukin- $1 \beta$ processing in monocytes. Nature $356: 768-774$.

Umemiya M, Raymond LA (1997) Dopaminergic modulation of excitatory postsynaptic currents in rat neostriatal neurons. J Neurophysiol $78: 1248-1255$.

Vickers NJ (2000) Mechanisms of animal navigation in odor plumes. Biol Bull 198:203-212.

Walsh PD (1996) Area-restricted search and the scale dependence of patch quality discrimination. J Theoretic Biol 183:351-361.

Ward S (1973) Chemotaxis by the nematode Caenorhabditis elegans: identification of attractants and analysis of the response by use of mutants. Proc Natl Acad Sci USA 70:817-821.

Weinshenker D, Garriga G, Thomas J (1995) Genetic and pharmacological analysis of neurotransmitters controlling egg laying in C. elegans. J Neurosci 15:6975-6985.

White J, Tobin TR, Bell WJ (1984) Local search in the housefly Musca domestica after feeding on sucrose. J Insect Physiol 30:477-487.

White JG, Southgate E, Thomson JN, Brenner S (1986) The structure of the nervous system of the nematode Caenorhabditis elegans. Philos Trans R Soc Lond B Biol Sci 314:1-340.

Wicks SR, Rankin CH (1997) Effects of tap withdrawal response habituation on other withdrawal behaviors: the localization of habituation in the nematode Caenorhabditis elegans. Behav Neurosci 111:342-353.

Yan Z, Hsieh-Wilson L, Feng J, Tomizawa K, Allen PB, Fienberg AA, Nairn AC, Greengard P (1999) Protein phosphatase 1 modulation of neostriatal AMPA channels: regulation by DARPP-32 and spinophilin. Nat Neurosci 2:13-17.

Zheng Y, Brockie PJ, Mellem JE, Madsen DM, Maricq AV (1999) Neuronal control of locomotion in C. elegans is modified by a dominant mutation in the GLR-1 ionotropic glutamate receptor. Neuron 24:347-361.

Zhuang X, Oosting RS, Jones SR, Gainetdinov RR, Miller GW, Caron MG, Hen R (2001) Hyperactivity and impaired response habituation in hyperdopaminergic mice. Proc Natl Acad Sci USA 98:1982-1987. 